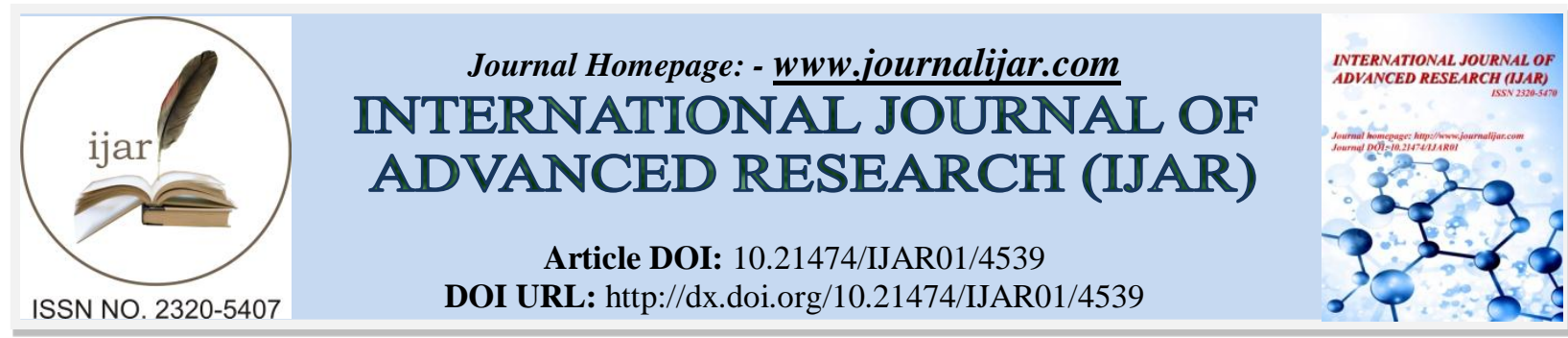

RESEARCH ARTICLE

\title{
FIRST LINE ANTI-MALARIAL DRUG (CHLOROQUINE) EFFICACY STUDY FOR THE TREATMENT
} OF PLASMODIUM VIVAX.

Dr.Shafiqullah Shafaq MD, MPH.

JSPH-Public health department, Maulana Azad University Jodhpur, Rajasthan, India.

\section{Manuscript Info}

\section{Manuscript History}

Received: 19 April 2017

Final Accepted: 21 May 2017

Published: June 2017

\section{Abstract}

Background: Malaria is well known parasitic disease and is a significant public health concern in Afghanistan. P. vivax, although usually considered to be benign, causes repeated debilitating relapses, rare life threatening complications, and low-birth-weight (LBW) infants of infected mothers. Antimalarial drug resistance poses a considerable challenge to malaria control, and is a major cause of malaria morbidity and mortality.

In Afghanistan, P. vivax is transmitted between the months of May and October, but also some rare cases till end of Dec and accounts for around $97.8 \%$ of malaria cases.

In Afghanistan malaria is the result more than $97 \%$ of plasmodium vivax and rarely plasmodium falciparum parasites, with focal and seasonal transmission and is a major health and wealth-threatening burden, now it is mostly confined to eastern region. Case management is major cornerstone of current control program and national treatment guideline recommends chloroquine as first line treatment regimens for vivax malaria respectively (eventually in combination with Primaquine for radical cure).

\section{Methods:}

This surveillance study was one-arm (descriptive) evaluation of clinical and parasitological responses to directly observed treatment with Chloroquine for $P$. vivax malaria of all male and female, nonpregnant female and children over six month. Treated on site with Chloroquine for P.vivax malaria and monitored for 28 days from Sep, 2016 to Feb, 2017 in eight location in eastern region (Nangarhar and Laghman provinces). All cases were followed up on days $0,2,7,14$, 21 and 28 for evaluation of post treatment clinical and parasitological responses. On the basis of the results of these assessments, the patients will be classified as having therapeutic failure (early or late) or an adequate response. The proportion of patients experiencing therapeutic failure during the follow-up period were used to estimate the efficacy of the study $\operatorname{drug}(\mathrm{s})$.

\section{Results}

192 Laboratory confirmed malaria patients (192 PV) were enrolled across eight health facilities of two provinces in the eastern part of the country. Baseline clinical and laboratory characteristics were at benign 
Pattern for inclusion in trial. Curative efficacy was highly perfect, with an adequate clinical and parasitological response (ACPR) of 99.5\% (191/192 treatment success) on day-28. Early therapeutic response of all patients treated with CQ the parasitological response was pleasant and better (within 48hours), mean different in temperature D2 vs D0 $=1.2 \mathrm{C}^{\circ}, \mathrm{p}<0.0001$. Parasitaemia: at day2 $7 / 133$ patients $(5 \%)$ remained $+\mathrm{ve}(\mathrm{p}$-value $<0.0001$

Gametocytes were found in $58 \% \mathrm{PV}$ at day- 0 and rapidly cleared with treatment as only $0.9 \%$ cases remained with gametocytes on day- 2 . Only 1/192 (0.5\%) recurrence cases found on day 28 .

Post treatment $\mathrm{Hb}$ recovery was also reassuring and there was significant improvement in Hb recovery (Day-28 vs. D-0 $=13.4$ vs. 12.50 (p-value <0.0006) 4

Drugs Safety: drug is well tolerated, no serious adverse event (SAE) or adverse event (AE) reported.

\section{Conclusions:}

The descriptive report of clinical outcomes of the study suggests good and high therapeutic efficacy of Chloroquine for vivax malaria treatment in Afghanistan as well as Primaquine for radical treatment. Due to its high efficacy, easy availability and broad use, we recommend to keep continue its administration in Afghanistan first line treatment of PV, but it is crucial to regularly monitor it's efficacy through clinical, laboratory (in-vitro) and genetic studies for molecular markers analysis.

Copy Right, IJAR, 2017,. All rights reserved.

Acronyms and Abbreviations:

\begin{tabular}{|c|c|}
\hline AADA & Agency for assistance and Development of Afghanistan \\
\hline ACT & Artesmisinin combination therapies \\
\hline AS & Artesunate \\
\hline BHC & Basic Health Center \\
\hline CHC & Chloroquine \\
\hline CQ & Comprehensive Health Center \\
\hline DH & District Hospital \\
\hline IRB & Institutional Review Board \\
\hline MRC & Nalaria and Research control center \\
\hline NMLCP & Plasmodium \\
\hline P & Plasmodium Falciparum \\
\hline PF & Primaquine \\
\hline PQ & Plasmodium Vivax \\
\hline PV & Sub Health Center \\
\hline RDT & Sulfadoxine-Pyrimethamine \\
\hline SHC & Vivax Treatment Afghanistan \\
\hline SP & World Health Organization \\
\hline VTA &
\end{tabular}




\section{Summary:-}

Title: First line anti-malarial drugs (Chloroquine) efficacy study for the treatment of Plasmodium vivax malaria in seven health facilities of Nangarhar and Laghman provinces of Afghanistan.

Purpose: to assess the efficacy of the current first line treatment policy.

Objective: To assess the efficacy of anti-malaria drugs (Chloroquine) for the treatment of Plasmodium vivax malaria infection.

Study Sites:-

1. Malaria Reference Center, Jalalabad, Nangarhar

2. Kama District Hospital, Nangarhar

3. Najmul Qura CHC, Nangarhar

4. Nahri Shahi BHC, Nangarhar

5. Sangar Sari CHC, Nangarhar

6. Beland Ghar CHC, Nangarhar

7. Najmul Jahad CHC, Nangarhar

8. Malaria Reference Center, Mehterlam, Laghman

Study Period:- Sep 2016 to February 2017

Study Design:- one-arm prospective study (Descriptive interventional study)

Patient population:- The population are consist of febrile patients aged above six months and confirmed $P$. vivax infection.

Sample Size:- The total of 200 patients were in plan in this study (20-25 PV cases for each site).

Treatment(s) and follow-up:- Chloroquine for $P$. vivax malaria.

Clinical and parasitological parameters were monitored over a 28 -day follow-up period to evaluate drug efficacy.

Primary endpoints:- The proportion of patients with early treatment failure, late clinical failure, late parasitological failure or an adequate clinical and parasitological response as indicators of efficacy.

\section{Secondary endpoints:-}

1. The frequency and nature of adverse events

2. Post treatment trend of hemoglobin,

\section{Introduction/Background:-}

Malaria is well known parasitic disease and is a significant public health concern in Afghanistan. Of the four species of malaria parasites that infect humans ( $P$. falciparum, $P$. vivax, $P$. malaria and $P$. oval), $P$. falciparum and $P$. vivax are the most common in the country. Plasmodium falciparum causes the most severe disease and almost all fatalities, whereas $P$. vivax, although usually considered to be benign, causes repeated debilitating relapses, rare life threatening complications, and low-birth-weight (LBW) infants of infected mothers . LBW also increases the risk of death in the newborn. Antimalarial drug resistance poses a considerable challenge to malaria control, and is a major cause of malaria morbidity and mortality.

In Afghanistan, $P$. vivax is transmitted between the months of May and October, but also some rare cases till end of Dec and accounts for around $97.8 \%$ of malaria cases.

Resistance to cheap and widely available drugs is documented throughout tropical regions. WHO recommends the use of Artesmisinin combination therapies to improve antimalarial effectiveness and keep the selection of drug resistant parasites to a minimum? Although changing antimalarial policy to Artesmisinin combination therapies are now well advocated, efforts continues for the most and long term effective combination and how these new treatments should be deployed and funded.

The Asian region, particularly neighboring Southeast Asia, has been a focus for emergence of drug resistant strains of $P$. falciparum to Chloroquine (CQ) for more than 50 years, followed by resistance to Sulfadoxine-Pyrimethamine $(\mathrm{SP})$ in the 1980s. However, drug resistance among $P$. vivax species has grown slower than among $P$ falciparum species, as the first CQ resistant isolates of $P$ vivax were reported from Papua, Indonesia, and Papua New Guinea and cases have also been reported from Myanmar, India and South America. 
In Afghanistan resistance of $P$. falciparum to CQ and Amodiaquine was first detected in 1989, and failure rates have increased to more than $60 \%$ for the country as a whole and as high as $90 \%$ for Jalalabad. In 2004 failure rates for SP for the treatment of $P$. falciparum was $10-15 \%$, while for vivax malaria CQ alone was effective till 2009 . The Ministry of Public Health, in consultation with WHO and other international partners, CQ continues as drug of choice for $P$. vivax mono-infection and $\mathrm{CQ}$ is the recommended treatment for patients with a presumptive diagnosis of malaria.

The Afghanistan NMLCP guideline also recommends that CQ can be continuous for the treatment of $P$. vivax malaria (eventually in combination with Primaquine for radical cure).

We proposed here one arm prospective study in which the efficacy and tolerability of CQ were assessed in patients with $P$. vivax infection. The follow-up was for 28 days of $P$. vivax studies [16]. The proposed study provided up-todate evidence on efficacy and safety of CQ for vivax infection in Afghanistan.

The results of this study will be used to assist the Ministry of Health of Afghanistan in assessing the current national treatment guidelines for P. Vivax malaria and to update the malaria treatment policy if necessary. 9

Since, the access to effective anti-malarial treatment is important cornerstone of malaria control, and WHO recommends conducting regular sentinels-based efficacy studies for monitoring of prevailed antimalarial drugs [10] in order to guide the development of national malaria treatment policy, the ministry of public health in collaboration with WHO, and independent researcher conducted this therapeutic efficacy and safety trial of WHO recommended fixed dose and presumed as effective Pv through discovering the emergence of resistance in its early stage and provide necessary data on better alternative and standby treatment regimen for policy-makers.

The present paper reviewed the in-vivo efficacy and safety of CQ for treating vivax and uncomplicated falciparum malaria. The paper present adequate clinical and parasitological response on day-28, parasite and fever clearance times, safety and tolerance profile in order to;

Provide update on country profile of fixed dose anti-malarial treatment regimens CQ widely available in local market, Simplify antimalarial treatment regimen

\section{Study Objectives:-}

The general objective of this study is to assess the therapeutic efficacy and safety of CQ for the treatment of P.vivax malaria in center of MRC Mehterlam district of Laghman and MRC center Nangarhar, Kama DH, Najmul Qura CHC, Beland Ghar CHC, Sangar Sari CHC, Najmul Jahad CHC and Nahri Shahi BHC in Nangarhar, Afghanistan.

\section{The primary objective is:-}

$\square$ To measure the clinical and parasitological efficacy of CQ in patients aged above six months suffering from vivax malaria, by determining the proportion of early treatment failure, late clinical failure, late parasitological failure or an adequate clinical and parasitological response as indicators of efficacy;

\section{The secondary objectives are:-}

$\square$ To evaluate the incidence of adverse events; and

\section{The optional exploratory objective is:-}

$\square$ To assess hemoglobin recovery after treatment with CQ

\section{Methods:-}

\section{Study Design:-}

This surveillance study was one-arm (descriptive) evaluation of clinical and parasitological responses to directly observed treatment with Chloroquine for $P$. vivax malaria. People with uncomplicated malaria who meet the study inclusion criteria were enrolled, treated on site with Chloroquine for P.vivax malaria and monitored for 28 days. The follow-up will consist of a fixed schedule of check-up visits and corresponding clinical and laboratory examinations. On the basis of the results of these assessments, the patients were classified as having therapeutic failure (early or late) or an adequate response. The proportion of patients experiencing therapeutic failure during the follow-up 
period were used to estimate the efficacy of the study drug(s). The study conducted between Sep/2016 -March/2017 10

\section{Study Sites:-}

The study conducted in 8 health facilities of (MRC Jalalabad, DH in Kama district, Najmul Qura CHC, Beland Ghar CHC in Behsood district, Sangar Sari in Kama district, Nahri Shahi BHC of Behsood district and Najmul Jahad CHC in Jalalabad city) Nangarhar province and health facility of (MRC) Laghman province under close supervision of malaria control centers formerly malaria reference centers (MRCs).

\section{Timing and duration of the study:-}

The study conducted during the malaria transmission season September, 2016, to February, 2017 to cover P. vivax transmission

\section{Study Population:-}

The study population were consist of patients with uncomplicated P.vivax malaria attending the study health clinic who is aged above 6 months. All adult patients signed a consent form for participation. Parents or guardians have given informed consent on behalf of children under 16 years of age. Children aged 15 years and older required to consent for participation by signing an informed assent form.

\section{Recruitment of patients:-}

All febrile cases as they presented to each health facility were screened and enrolled according the eligibility criteria (age $>6$ months, microscopic detected malaria infection, history of fever in previous $24 \mathrm{~h}$ or axillary temperature $>37.5$, provision of informed consent, absence of: danger signs \& severity, other reason for fever, previous anti-malarial taken within one week, pregnancy or lactation).

\section{Treatment and follow-up:-}

Demographic information, vital signs and findings of clinical examination, complete medical history and prior medication were documented, including body weight and temperature measured in axillary (or orally) by thermometer. Blood samples were taken for $\mathrm{Hb}$ and WBC measurement as well as preparation of thick and thin films with Giemsa-staining to examine and count parasitaemia (both sexual and asexual forms) by WHO recommended method (counting against $200 \mathrm{WBC}$ in thick film with estimation of $8000 \mathrm{WBC} / \mu \mathrm{L}$ ). Slides were cross-checked externally by reliable microscopists.

From each subject three drops of blood were collected at enrolment for estimating genetic background of parasite species, accuracy of microscopic diagnosis, PCR analysis, detection of resistance markers, one drop on day-7 for estimating drug level in blood and only from recurred cases another three drops were collected on day of recurrence for paired examination of PCR analysis in order to differentiate recrudescence from reinfection. All these samples were collected on a filter papers (Whitman $3 \mathrm{M}$ ) and kept in room temperature and later transferred to Oxford Mahidol University for analysis.

Daily direct observed (by clinician or health worker) doses of CQ were administered orally according to weight bands using standard dose of $10 \mathrm{mg} / \mathrm{kg}$ for first and second day and $5 \mathrm{mg} / \mathrm{kg} 11$

on third day to all patients and patients were kept for 30 minutes after receiving the medication, in order to repeat dose if they vomit.

After day- 0 visit all subjects were seen for day- 2 for clinical and laboratory assessment and to complete observed treatment and then seen on weekly base and clinically as well as laboratory check-up done as per approved case record forms till day-28 (Day 0, 2, 7, 14, 21, 28,) in order to detect recurrences.

\section{Patient discontinuation or protocol violation:-}

Study patients who meet any of the following criteria were classified as withdrawn.

- Withdrawal of consent. All the patients withdrawn consent, who didn't want to further follow-up or treatment at the study site.

- Failure to complete treatment: All the enrolled patients complete the treatment without failure. 
- There were three patients missed the second visit on day2.

- There was no any serious adverse events recorded in order to terminate the follow up visits.

\section{Sample size:-}

The treatment failure rate to CQ in the area is assumed to be 5\%. At a confidence level of $95 \%$ and a precision around the estimate of 3-4\%, a minimum of 200 patients were included. With a $20 \%$ increase loss to follow-up and withdrawals during 28-days follow-up period, 20-25 patients were planned to be included in the study per test drug and per site.

\section{Study Outcome:-}

Primary out-come for the study was overall treatment success Out-comes were classified according WHO guide-line as early therapeutic response (parasite, fever clearance times and symptoms recovery) and late therapeutic (clinical or parasitological) response (cumulative cure rate day-28), loss to follow-up, withdrawal from study and Adequate clinical and parasitological response (ACPR). The secondary outcomes were Gametocytemia, post treatment $\mathrm{Hb}$ level and adverse events

\section{Data management and statistical analysis:}

The principal investigator ensured that the study protocol is strictly adhered to and that all data are collected and recorded correctly on the case report form. Laboratory and clinical data recorded on a daily basis on the case report form designed for the study. Data derived from source documents and consistent with the source documents. All case report forms were checked for completeness.

After the study completeness, the data were entered into an excel sheet which was designated according to the objective of the study and the study data stored in a computer for maintaining confidentiality. 12

The principal investigator is responsible for keeping all screening forms, the case report form and the completed subject identification code list in a secure location.

The data analysis excel sheet was used for data management and analysis according to the study outcomes.

A description of baseline characteristics of all patients enrolled in study was presented. The Kaplan Meier method was used to measure the efficacy of CQ by calculating the cumulative risk of failure at day-28. There was comparison of parasite and study sites on: proportion of lost to follow up, ACPR, post treatment Hb level.

During the study period we could abled to find 192 PV cases (58\% male) according to the inclusion criteria and most of the cases were $<18$ years children.

Only PV case (1/192) had nausea and vomiting in this trail, therefore the efficacy of the CQ was around 99.5\%.

We had 37/192 case (19\%) missed to follow up the visits and they have withdrawn from the study due to many reasons.

\section{Ethical considerations:-}

The study received official approval from Institutional review board (IRB) of afghan national public health institute, ministry of public health.

\section{Result:-}

Recruitment:-

From Sep, 2016 to End of Feb, 2017, 192 Plasmodium Vivax positive patients were enrolled across the eight study centers of two provinces named Nangarhar and Laghman in eastern part of Afghanistan. 
Table 1:- shows the baseline characteristics of enrolled patients:

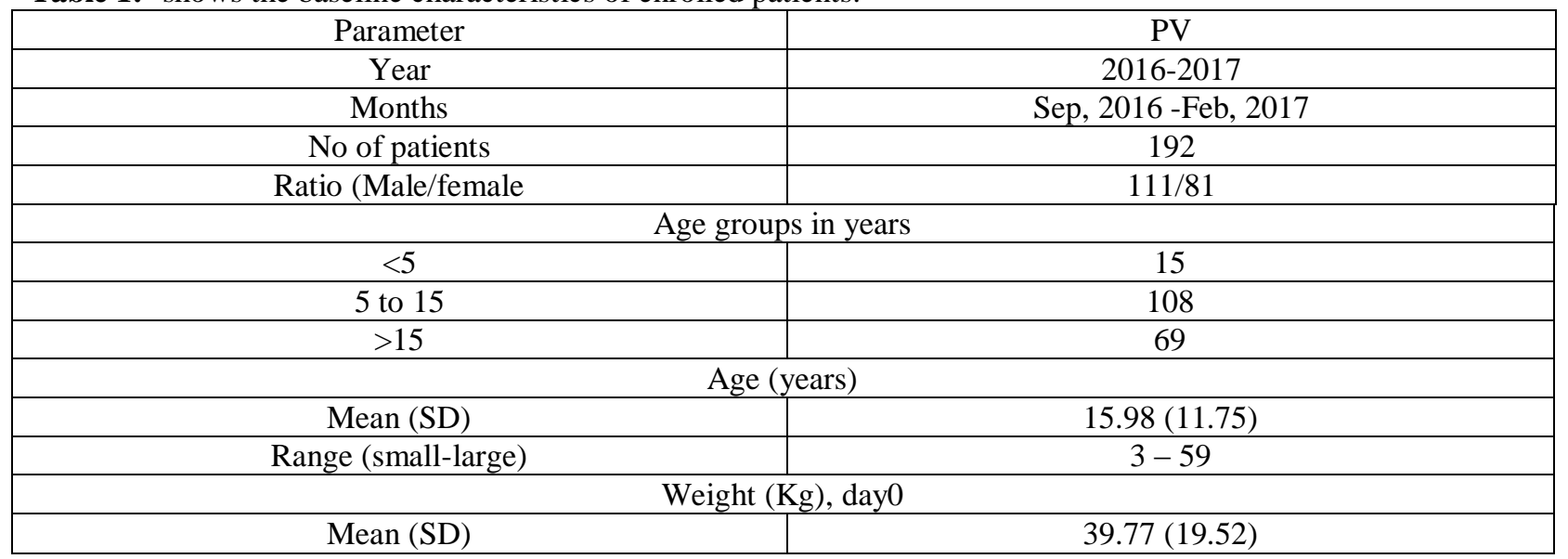

\section{Clinical and laboratory findings on admission:-}

The clinical findings such as vital signs (pulse, respiratory rate, blood pressure), and laboratory findings particularly $\mathrm{Hb}$ and WBC measurement were relatively normal in all patients. The disease feature in enrolled patients was mild or moderate indicating the benign pattern of enrolled patients as inclusion criteria.

The overall parasitaemia (asexual forms) ranged from 180-40800/ $\mu \mathrm{L}$ and parasitaemia Geometric mean (SD) 2557 (5789) with around $58 \%$ proportion of patients with enrolment gametocytemia.

Overall on D0 $9.8 \mathrm{mg} / \mathrm{kg} \mathrm{CQ}$ is administered with available tab of $150 \mathrm{mg}$ base CQ.

Table 2:- Clinical and Laboratory details of patients on admission for CQ study

\begin{tabular}{|l|l|l|}
\hline SN & Parameter & PV \\
\hline 1 & Temperature $\left({ }^{\circ} \mathrm{C}\right)$ day0 & \\
\hline \multirow{2}{*}{2} & Mean $(\mathrm{Sd})$ & $37.5(0.74)$ \\
\cline { 2 - 3 } & Range $($ small-large) & $35.1-41$ \\
\hline \multirow{3}{*}{3} & Asexual Parasitaemia $(\mu \mathrm{L}), \mathrm{D}-0$ & \\
\cline { 2 - 3 } & Geometric Mean $(95 \% \mathrm{CI})$ & $2557(5789)$ \\
\cline { 2 - 3 } & Range (small- Large) & $180-40800$ \\
\cline { 2 - 3 } & Gametocytemia \% & 58 \\
\hline \multirow{2}{*}{} & Total drug dose $(\mathrm{mg} / \mathrm{kg})$ & $9.8(1.14)$ \\
\hline & Mean $(\mathrm{Sd})$ & $150-600$ \\
\cline { 2 - 3 } & Range $($ small-Large) & \\
\hline
\end{tabular}

\section{Treatment Outcome:-}

Early therapeutic response:-

Chloroquine (25mg/kg bw) divided into 3 days (day1-10mg/kg, day2-10mg/kg and day $3-5 \mathrm{mg} / \mathrm{kg}$ ) are administered to patients with uncomplicated P. vivax malaria. The correct drug dosage are determined from the dosing chart (Appendix 3).Tablets of Chloroquine (Resochin DS) (150 mg base) available in the HFs.

Early therapeutic response of all patients treated with CQ the parasitological response was pleasant and better (within 48hours), significantly more patients cleared their asexual parasites. This parasites clearance at day-1 was $44 \%$ in vivax, at day-2 the parasite clearance was $94 \%$.

The proportion of patients with body temperature $\geq 37.5^{\circ} \mathrm{C}$ was $104 / 192(54 \%)$ at enrolment, in day-2 post treatment only $4 \%$ remained with body temperature $\geq 37.5^{\circ} \mathrm{C} .14$

Long - Term therapeutic Efficacy (ACPR);-

The Long term Efficacy was perfect (99.3\% ACPR on day-28) in PV ACPR rate, only one patient $(0.75 \%)$ on day28 had parasites in the blood. 
Graph 1:- Shows CQ administration success for PV

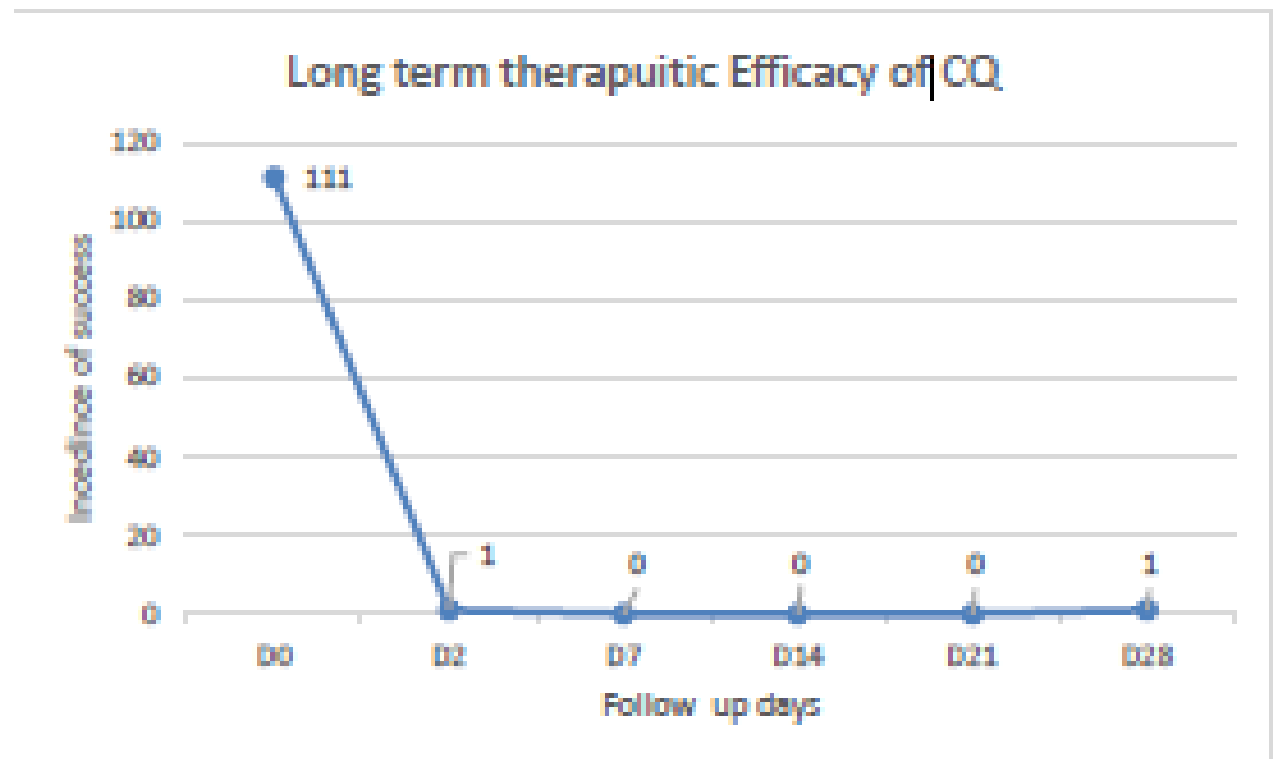

\section{Other Laboratory Findings:-}

Hemoglobin measurement performed on day of enrolment and all follow up visits, on enrolment day Hb mean (SD) was $12.5(2) \mathrm{vs} \mathrm{Hb}$ mean (SD) 13.2 (1.5) with 5.6\% improvement from the day of enrolment. At enrolment day anemic $(\mathrm{Hb}<10 \mathrm{~g} / \mathrm{dl})$ were mainly in $<10$ years children. Post treatment $\mathrm{Hb}$ recovery was reassuring similarly as only $4 \%$ (one patient) remained anemic by day- 28 .

Clinical recovery following treatment occurred in all cases successfully during 48 hours

\section{Gametocyte Carriage:-}

Gametocytes were found in 111/192 PV cases at day-0 and rapidly cleared with treatment as $1 / 111$ PV case remained with gametocytes on day-2. In weekly follow-up visits $1 / 192 \mathrm{PV}$ at day-28 was newly found with gametocytes carriage.

\section{Occurrence of Adverse Events:-}

Fortunately, there was no severe adverse event observed in the study. In term of mild adverse events only one PV case observed with vomiting \& headache at day-2. 15

\section{Discussion:-}

The study showed that the overall efficacy of CQ was high (99.5\%) in Nangarhar and Laghman province where vivax species cause morbidity. This high efficacy despite its wide and irrational use particularly in private sector is reassuring. Comparing D-0 with D-2 the signed and septum are changed in the patients. On D-2 only eight cases $(8 / 104>$ or $=37.5 c)$ had fever. Only one nausea and omitting case on day- 2 .

There was a dramatic reduction in parasitaemia in the study participants after two day's dose of CQ. This rapid parasite clearance potential contributes to a quick relief of symptoms, a decrease in risk of anaemia, reduction of gametocytes carriage rate and transmission potential.

From all the above mentioned characteristics the early therapeutic response by administration of CQ was successfully.

This extended follow up study corresponds with other studies done in other countries of Africa on the efficacy of AL [12],[13],[14],[15] and further benefited the assurance of efficacy up to day-28. The study showed that there was only one treatment failure, although differentiation between recurrence and recrudescence of the initial infection from re-infection is still due and helpful PCR analysis is awaited. The differentiation is of particular concern in areas 
with high vivax transmission resulting in high relapses proportion but use of an anti-malarial drug with longer halflife to suggest better suppressive activity for preventing relapse and re-infection might be a target for drug resistance development. Treatment failure can be influenced by several factors more often a decrease in drug concentrations [16], therefore collection blood samples at day-7 was part of this study and the result is awaited.

The limitation of this study were the under-power and unavailability of enough sample and control group to allow comparison and subgroups analysis between sex \& age groups, urban and rural settings, study sites and so on, in order to undertake detailed assessment to discover and clarify demographic and epidemiological reasons associated with treatment failures.

It is possible that the action of AL (Artemether-Lumefantherine) is simply to delay relapses of vivax malaria rather than prevent them. Studies with longer-term follow-up would be needed to determine if this is the case and if AL offers tangible benefits in terms of long-term health of the individual (preventing relapse and associated anaemia) and possibly the community (by reducing transmission). Any health benefits have to be balanced against the relative cost of treatment course, which is currently in favor of standard treatment regimen. These issues also have to be considered in the context of the challenges of treating patients with radical therapy in this region

The recommendations for the WHO malaria treatment guidelines in the year 2010 emphasizes on switching to another anti-malarial drug when the first-line has more than a $10 \%$ overall failure rate [9]. Therefore, national malaria control program with collaboration of partners should continue monitoring of standard first-line drug's efficacy and resistance in sentinel surveillance sites.

\section{Conclusion:-}

The findings of the study showed that the CQ has high efficacy in eastern of Afghanistan. Therefore, it is important to monitor efficacy in order to detect and prevent resistance of CQ as it is widely used in private sector. Surveillance studies for monitoring of standard treatment should include such widely used drugs. There is also need to further investigate the comparatively efficacy rate in order to identify possible determinants of treatment failure. Consistent with the known pharmacological properties of the drugs, CQ provides a longer period of post-treatment prophylaxis, reducing the incidence of recurrence, can be easily implemented in current health system (BPHS), therefor it should be kept continue for the treatment of PV cases management in Afghanistan according to the NMLCP guideline.

\section{Acknowledgements:-}

This study was support by my own self and AADA as consultant. We are grateful to the doctors, Laboratory technicians and malaria workers in the study sites, implementing NGO AADA as well as PPHDs and the senior management. Especially thanks from my guider who gave me plenty support for accomplishment of this trial. 16

\section{Contributions:-}

This project was designed by me and my co-guide and we were directly responsible for supervision and monitoring of field work and sample collection. The paper was drafted by me and reviewed by my co- guide and approved the final version.

\section{References:-}

1. MoPH. National Malaria Strategic Plan 2012-2016.

2. NMLCP. 2013 annual HMIS report.

3. National Malaria treatment guideline 2014

4. Global Malaria report 2016

5. Antimalarial therapeutic efficacy:

6. Awab GR, et al. Dihydroartemisinin-piperaquine versus Chloroquine to treat vivax malaria in Afghanistan: an open randomized, non-inferiority trial. Malar J2010; 9:105.

7. Awab GR, et al. Prevalence of antifolate resistance mutation in Plasmodium falciparum isolates in Afghanistan. Malar J 2013; 12:96.

8. Baird JK. Chloroquine resistance in Plasmodium vivax. Antimicrob Agents Chemother 2004; 48:4075-83.

9. WHO. Global Report on Antimalarial Drug Efficacy and Drug Resistance 2000-2010. Geneva, Switzerland: World Health Organization 2010. 
10. Durani Net.al. Efficacy of combination therapy with artesunate plus Amodiaquine compared to monotherapy with Chloroquine, Amodiaquine, or SP for treatment of uncomplicated Plasmodium falciparum in Afghanistan. Trop Med Int Health. 2005 Jun; 10(6):521-9.

11. Malaria control and elimination: WHO. Guideline for treatment of malaria. 3rd edition. Geneva, Switzerland: WHO 2015.

12. Clinical trials for assessment therapeutic efficacy

13. WHO. Method for surveillance of antimalarial drug efficacy. Geneva, Switzerland: WHO 2009.

14. Health promotion and education Research methods (textbook)

15. Clinical trials in context of biomedical ethics ICH-GCP E6; http://www.ich.org/.... 17 Declaration of Helsinki: http://www.wma.net/30...

16. WHO (2010) Guidelines for the treatment of malaria (2nd edition): WHO.

17. Jamornthanyawat N, Awab GR, Tanomsing N, Pukrittayakamee S, Yamin F, et al. (2014) A Population Survey of the Glucose-6-Phosphate Dehydrogenase (G6PD) 563C.T (Mediterranean) Mutation in Afghanistan. PLoS ONE 9(2): e88605. doi:10.1371/journal.pone.0088605. 